\title{
Aldosterone, hypertension and heart failure: insights from clinical trials
}

\begin{abstract}
John W Funder
Two clinical trials will be reviewed, RALES $^{1}$ and ILLUMINATE. ${ }^{2}$ In RALES, low-dose spironolactone in addition to standard of care, produced a $30 \%$ improvement in survival in progressive heart failure, commonly assumed to reflect deleterious effects of aldosterone, with spironolactone competing with aldosterone for cardiac mineralocorticoid receptors. Recent evidence, however, points to cortisol rather than aldosterone as the hormone activating cardiac mineralocorticoid receptors, under conditions of tissue damage, and spironolactone as acting by mechanisms other than receptor blockade. ILLUMINATE compared the effects of torcetrapib, a cholesterol ester transport protein inhibitor, in combination with atorvastatin vs. atorvastatin alone, and was terminated after excess mortality was found in the torcetrapib arm. Subjects receiving torcetrapib showed effects consistent with increased aldosterone secretion, subsequently confirmed on patient samples and in vitro. In animal experiments, the pressor effect of torcetrapib was abolished by adrenalectomy but not by administration of trilostane, an inhibitor of aldosterone secretion. Although aldosterone (and probably cortisol) excess is involved in the off-target effects of torcetrapib, they may also involve secretion of endogenous oubain from the adrenal glomerulosa. This possibility may explain the enigma of aldosterone being homeostatic in chronic sodium deficiency, but deleterious in the presence of inappropriate sodium levels. Hypertension Research (2010) 33, 872-875; doi:10.1038/hr.2010.115; published online 15 July 2010
\end{abstract}

Keywords: aldosterone; clinical trials; mineralocorticoid receptors; spironolactone; torcetrapib

\section{INTRODUCTION}

Translational research is often given an unidirectional sense, 'from benchtop to bedside'. Before the ascendancy of cellular and molecular biology insights into mechanisms of disease (and in turn, of normal physiology) often were the result of astute clinical observation and analysis of 'experiments of nature'. In the current era of powerful techniques available to the laboratory investigator, clinical trials are primarily judged on their outcomes and any adverse/off-target effects, with scant attention paid to the possibility that they may prompt often radical reconsideration of the basic biology involved.

Of the two trials analyzed in this review, one (RALES: Randomized Aldactone Evaluation Study $)^{1}$ was judged as an overwhelming success, in terms of improvement in survival (30\%) and morbidity (35\%) when low-dose $\left(\overline{\mathrm{x}} 26 \mathrm{mg} \mathrm{day}^{-1}\right)$ spironolactone in addition to standard of care was given to patients with progressive heart failure. It might also be judged a success in that a 10-fold higher incidence of gynecomastia, even at the low dose used, prompted the development of the selective mineralocorticoid receptor (MR) antagonist eplerenone. It might even be argued that its obligate side-effect (not an offtarget effect) of an increase in mean plasma $\left[\mathrm{K}^{+}\right]$of $0.2-0.3 \mathrm{mmoll}^{-1}$ was a success, in that it has subsequently prompted a number of companies to develop lead compounds of tubule-sparing (that is, tissue selective) MR antagonists, to mitigate the risk of hyperkalemia.
The second trial to be analyzed (ILLUMINATE: Investigation of Lipid Level Management to Understand the Impact on Atherosclerotic Events), ${ }^{2}$ in contrast had to be terminated early due to a clear increase in mortality in the arm including torcetrapib, the compound under test. Torcetrapib is a powerful inhibitor of cholesterol ester transport protein, repeatedly shown to raise plasma levels of HDL and to lower those of LDL. ILLUMINATE was a major outcome trial on over 15000 at-risk subjects, comparing torcetrapib plus atorvastatin ('Lipitor') with atorvastatin alone. The reason for termination was that addition of torcetrapib, despite its expected effects on HDL and LDL, not only showed no benefit compared with atorvastatin alone, but in fact resulted in increased all-cause mortality. On both criteria, then-non-superiority, and off-target effects-ILLUMINATE must be judged a failure.

The connection between RALES and ILLUMINATE, and thus the point of yoking them as the subject of this review, is not immediately obvious. The connection is that the beneficial effects found in RALES are commonly ascribed to spironolactone blocking aldosterone from its adverse effects on cardiomyocytes (which is almost certainly wrong), and the off-target effects of torcetrapib to its stimulating aldosterone secretion (which is almost certainly correct, but only a partial explanation, on two counts). The point of considering the two together is that strictly from the viewpoint of basic biology it does not matter whether a clinical trial is a success or not; all are worth 
prospecting and possibly mining for the insights they may provide; translational research goes both ways.

\section{RALES}

There are two enigmas in RALES. The first is that-despite standard of care, which in some patients included diuretics-mean plasma levels of aldosterone were in the low normal range, and sodium status unremarkable. The second is the low dose of spironolactone $\left(\overline{\mathrm{x}} 26 \mathrm{mg} \mathrm{day}^{-1}\right.$ ) found to be adequate to produce the remarkable effects on morbidity and mortality that it did.

In terms of the first of these enigmas, it is clear that elevation of plasma aldosterone per se can have deleterious cardiovascular effects independent of blood pressure elevation. In patients with modestly raised plasma aldosterone levels due to glucocorticoid remediable aldosteronism, but with normal blood pressure, clear signs of cardiovascular damage have been demonstrated compared with age-, sexand blood pressure-matched controls. ${ }^{3}$ In patients with primary aldosteronism and substantially elevated levels of plasma aldosterone, a much higher prevalence of atrial fibrillation, stroke and myocardial infarction has been shown compared with age-, sex- and blood pressure-matched essential hypertensives. ${ }^{4}$ What this may be reasonably taken as showing is that even modest elevation of plasma aldosterone may have deleterious effects on the cardiovascular system. Added to this, from longitudinal survey data, there is a positive correlation between progressive increase in blood pressure over time and plasma aldosterone concentrations, even when the latter are in the normal range. ${ }^{5}$

At a stretch, then, it may be postulated that aldosterone is responsible for the activation of cardiac mineralocorticoid receptors, even given the low normal plasma levels in RALES. There is, however, experimental evidence and good reason to believe that the culprit in terms of MR activation in RALES is cortisol. To make this case a brief excursion into the mechanisms of selective activation of MR is necessary, as follows. Aldosterone circulates at levels $\sim 1 / 1000$ those of the physiological glucocorticoid cortisol (corticosterone in rats and mice), but is only $\sim 50 \%$ bound to plasma protein, compared with $95 \%$ for the glucocorticoids, so that plasma-free levels of aldosterone are $\sim 1 \%$ those of cortisol. Across a range of species MR have equivalent high affinity for cortisol as for aldosterone, raising the question of how aldosterone can act selectively at epithelia to modulate sodium balance. The answer lies in the co-expression in epithelia of the enzyme $11 \beta$ hydroxysteroid dehydrogenase Type 2 (11ßHSD2), for which aldosterone is not a substrate, which debulks intracellular cortisol by a factor of $90 \%$, converting it to receptorinactive cortisone. ${ }^{6,7}$ This still leaves 10 -fold higher intracellular levels of glucocorticoid than of aldosterone to bid for MR occupancy but not, under normal circumstances, to act as an MR agonist. ${ }^{8}$

When $11 \beta$ HSD 2 is congenitally deficient, or blocked as in licorice abuse, cortisol clearly acts as an MR agonist. This is unlikely to be due to intracellular cortisol levels going from 10 to 30 or 100 -fold those of aldosterone, and there is evidence that this reflects the changes in cellular redox state when $11 \beta \mathrm{HSD} 2$ is inoperant. For every molecule of cortisol converted to cortisone, a molecule of the obligate cosubstrate $\mathrm{NAD}$ is converted to NADH. The mechanism whereby NADH holds glucocorticoid-MR complexes inactive is yet to be determined, though similar mechanisms involving NADH have been described for other transcription factors. ${ }^{9}$ The vessel wall is an aldosterone target tissue, in that it coexpresses MR and 11ßHSD2; the actions of aldosterone are mimicked by cortisol in the presence, but not the absence, of carbenoxolone to block the action of $11 \beta \mathrm{HSD} 2 .{ }^{10}$ Cardiomyocytes, in contrast, do not co-express 11ßHSD2 with MR, so that in this tissue
MR are normally overwhelmingly glucocorticoid-occupied-but not activated..$^{11}$

In the failing heart, as in RALES, or post-myocardial infarction, as in EPHESUS, ${ }^{12}$ cardiomyocytes show high levels of reactive oxygen species, with accompanying changes in intracellular redox state. In ischemia-reperfusion studies, ${ }^{13}$ aldosterone increases area at risk and infarct size in Langendorf (isolated, perfused) preparations of rat hearts. In the same preparations, cortisol-at low nanomolar dosesmimics these aldosterone effects; the effects of aldosterone are blocked by spironolactone and those of cortisol similarly by spironolactone but not by the glucocorticoid receptor/progesterone receptor antagonist RU486. Given this demonstration of the MR agonist activity of cortisol in the context of tissue damage, the first of the enigmas of RALES (low normal aldosterone levels) appears to be answered; the predominant MR agonist in the context of cardiomyocyte damage is cortisol, not aldosterone.

Similar studies in the Langendorf ischemia-reperfusion model have thrown light on the second of the enigmas posed by the RALES trial, that of the efficacy of the very modest dose of spironolactone. Spironolactone not only antagonizes the effects of aldosterone and cortisol, on area-at-risk and apoptotic index, but administered alone is protective-that is, it reduces the area at risk and apoptotic index below control (no added steroid) levels. ${ }^{13}$ It does this in hearts from both intact and adrenalectomized rats, and at low nanomolar concentrations, by reciprocal actions on key effectors in the apoptotic pathways (AS Mihailidou, unpublished). There is no question that at therapeutic concentrations spironolactone might substantially exclude aldosterone from MR, although given its much higher intracellular levels, excluding cortisol is another question altogether. The demonstration of the cardioprotective effect of spironolactone, at low nanomolar concentrations absent any other steroid, is evidence that its predominant action is via induction of anti-apoptotic/repression of pro-apoptotic gene expression, at concentrations at which only a minority of MR would be spironolactone-occupied. This effect of spironolactone, to act as an inverse agonist at MR rather than merely by excluding agonists, thus provides an explanation for the second of the enigmas posed by RALES, that of the low spironolactone dose producing such a remarkably protective effect.

\section{ILLUMINATE}

Subjects given torcetrapib in addition to atorvastatin showed the anticipated changes in HDL and LDL levels compared with those receiving atorvastatin alone. In addition, they showed highly significant increases in blood pressure (systolic $4 \mathrm{~mm} \mathrm{Hg}$, diastolic $2 \mathrm{~mm} \mathrm{Hg}$ ), plus small but again highly significant elevations in serum $\left[\mathrm{Na}^{+}\right]\left(0.58 \mathrm{mmoll}^{-1}\right)$ and $\left[\mathrm{HCO}_{3}{ }^{-}\right]\left(0.27 \mathrm{mmoll}^{-1}\right)$ in the torcetrapib arm, and a decrease in serum $\left[\mathrm{K}^{+}\right]\left(0.14 \mathrm{mmoll}^{-1}\right)$. In line with these changes, post hoc analysis of plasma samples showed similarly small but significant increases in plasma aldosterone concentrations in the torcetrapib group. The default explanation for the increased mortality in the torcetrapib group is thus that of its off-target effect of elevating plasma aldosterone concentrations in a high risk, event-susceptible population, notwithstanding the relatively modest magnitude and duration of exposure. The effect of torcetrapib to produce sustained elevation of aldosterone secretion has been subsequently confirmed by a number of in vivo and in vitro studies. ${ }^{14-16}$

There are two issues, one buried in the trial data and the other from subsequent in vitro studies, that suggest that the default explanation may not be the whole story. In this cohort of over 15000 subjects, the risk ratio for 'any cause of cardiovascular' death was 1.4:1 (49 vs. 35 
patients). In contrast, the risk ratio for 'any non-cardiovascular cause of death' was 2:1 (40 vs. 20 patients); absolutely and relatively the relative risk is clearly higher in the non-cardiovascular group. Discussion has appropriately focused on aldosterone in the cardiovascular group, but until recently ${ }^{17}$ no consideration given to the 'any noncardiovascular' group. From in vitro studies it is clear that torcetrapib elevates both aldosterone and glucocorticoid secretion from the adrenal cortex. Of the excess deaths in the non-cardiovascular group, 19 of the 20 comprised cancer (24-14) and infection (9:0, all in diabetic patients). This is suggestive that increased cortisol secretion might possibly be affecting immune surveillance of occult neoplasia in a cohort containing many elderly patients, and on the outcomes of intercurrent infection in diabetic patients.

In terms of the 'any cause cardiovascular' deaths, the default explanation holds - but a series of subsequent in vitro and in vivo animal studies suggest that it may be a partial rather than the total explanation of the clinical outcome seen. Studies by Forrest et al. ${ }^{14}$ showed that in vivo torcetrapib stimulated aldosterone secretion and raised blood pressure, and $\mathrm{Hu}$ et al. ${ }^{15}$ showed that corticosteroid release correlated with pressor activity across a series of cholesterol ester transport protein inhibitors in rats and non-human primates. Blood pressure elevation does not appear to be a direct effect on the vasculature, in that torcetrapib did not increase perfusion pressure in the isolated perfused rat hind limb preparation. ${ }^{14}$ It also raised blood pressure in pithed rats, evidence against a primarily central mechanism and no attenuation of the pressor response was seen in dogs pretreated with prazosin or atenolol. ${ }^{14}$

What raises the index of suspicion are studies on adrenalectomized rats and intact rats treated with the $3 \beta$ hydroxysteroid inhibitor trilostane. ${ }^{14}$ Consistent with a primary role for aldosterone in blood pressure elevation in these animals is that adrenalectomy abolished the pressor response to torcetrapib. Not consistent with at least an acute pressor role for aldosterone is that blockade of steroidogenesis in vivo by trilostane administration abolished the increase in aldosterone levels in response to torcetrapib, but did not impair the pressor response. A logical interpretation of these observations is that in addition to corticosteroids (which are not responsible for this acute pressor response) torcetrapib is elevating the secretion from the adrenal of a non-catechol agent, which raises blood pressure, at least acutely.

One possible such agent is endogenous ouabain (EO), known to be secreted from the adrenal zona glomerulosa in response to ACTH, to angiotensin II via $\mathrm{AT}_{2}$ receptors, and to chronic sodium loading. ${ }^{18}$ Studies from the Blaustein laboratory, ${ }^{19}$ on wild-type and mice heterozygous $(+/-)$ for null mutations in the genes coding for the $\alpha 1$ or $\alpha 2$ subunit of the $\mathrm{Na}^{+}$pump, have shown that ouabain raises blood pressure and myogenic tone by reducing $\alpha 2$ subunit $\mathrm{Na}^{+}$pump activity and enhancing $\mathrm{Ca}^{++}$entry via the $\mathrm{Na}^{+} / \mathrm{Ca}^{++}$exchanger. In parallel studies from the Lingrel laboratory ${ }^{20}$ the $\alpha 2$-subunit gene sequence was minimally mutated to make the protein ouabainresistant. In contrast with wild-type mice, which show progressive elevation of blood pressure to a plateau $25 \mathrm{~mm} \mathrm{Hg}$ above control over 4 days, mutant mice show no elevation in blood pressure in response to ACTH. In wild-type mice, infusion of Digibind (GlaxoSmithKline, London, UK) abolished ACTH-induced hypertension, as did KB-R7943 (Tocris Bioscience, Ellisville, MO, USA), which inhibits the reverse mode of the $\mathrm{Na}^{+} / \mathrm{Ca}^{++}$exchanger. Studies are currently in progress to determine if torcetrapib stimulates the release of EO from the adrenal cortex, and additional studies planned to examine whether (or not) it raises BP in ouabainresistant mice.
Response of aldosterone and (putatively) endogenous ouabain secretion to torcetrapib

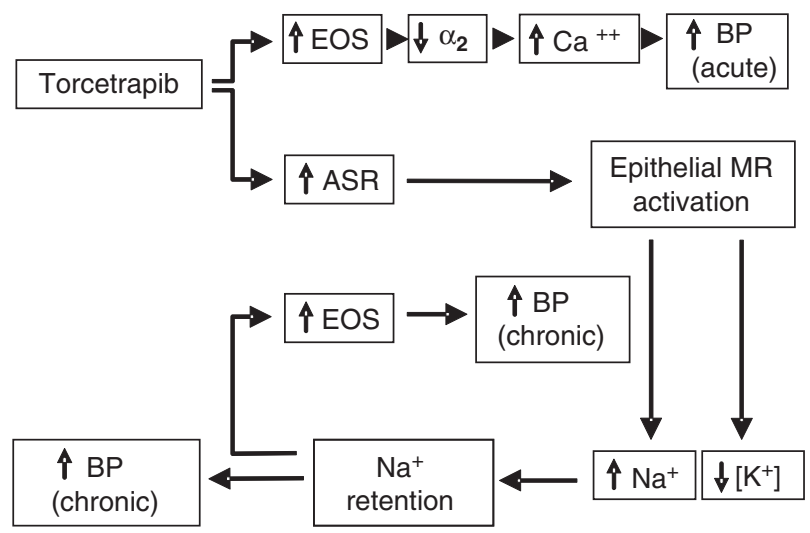

Figure 1 Interaction between aldosterone and endogenous ouabain in chronic blood pressure elevation, and putatively in acute torcetrapib-induced elevation of blood pressure.

Endogenous ouabain is obviously not the only non-corticosteroid, non-catechol adrenal secretion product, but it now has a well defined and both acute and sustained (for at least 4 days) pressor action on the vasculature. Physiologically, in terms of homeostasis, elevation of EO secretion in response to $\mathrm{Na}^{+}$loading may be to raise blood pressure and thus dump sodium via a pressure natriuresis. If torcetrapib in fact is shown to elevate EO secretion from the adrenal cortex, it may thus be that it forms part of a positive feedback loop with the undisputed increase in aldosterone secretion, with EO secretion supported chronically not only by torcetrapib but also by the aldosteroneinduced positive sodium balance (Figure 1).

The possible inferences for the pathophysiology of aldosterone are exciting, particularly given that classical MR antagonists (for example, canrenone) are commonly used in studies on EO as partial agonists/ predominant antagonists. In chronic $\mathrm{Na}^{+}$deficiency, aldosterone levels can be extremely high-consistent with occupancy of unprotected cardiac MR substantially above normal levels-with no elevation of blood pressure or deleterious cardiovascular sequelae; under such conditions aldosterone is clearly homeostatic. When, however, aldosterone levels are inappropriate for sodium status, as in primary aldosteronism clinically, or in mineralocorticoid/salt animal models, even modestly elevated aldosterone levels have deleterious rather than homeostatic effects. The difference may be that in chronic $\mathrm{Na}^{+}$ deficiency EO secretion is suppressed, whereas with $\mathrm{Na}^{+}$loading it is elevated. This reciprocity, in terms of response to $\mathrm{Na}^{+}$status, may provide the key to a persisting enigma in aldosterone $/ \mathrm{Na}^{+}$pathophysiology - that of why aldosterone levels inappropriate for sodium status can be so detrimental in the cardiovascular system, whereas much higher levels in chronic sodium deficiency are not damaging but homeostatic. It may be that the enigma posed by this off-target effect of torcetrapib, on 'any cause cardiovascular mortality', may shed light on a more basic - and much older-enigma, that of how sodium status stochastically modifies target tissue responses to MR activation by aldosterone.

\section{CONCLUSION}

Clinical trials are designed with important but limited criteria in terms of outcomes, those of efficacy and safety. Buried in many clinical trials-RALES, ILLUMINATE and others ${ }^{21}$ —are data which may have profound implications for clinical physiology and basic biology. Such 
rich seams of data need to be systematically mined, and research needs to be translated in both ways; not just bench top to bedside, but for clinical insights to inform and focus basic biological investigation.

1 Pitt B, Zannad F, Remme WJ, Cody R, Castaigne A, Perez A, Palensky J, Wittes J. The effect of spironolactone on morbidity and mortality in patients with severe heart failure. N Engl J Med 1999; 10: 709-717.

2 Barter P, Caulfield M, Eriksson M, Grundy SM, Kastelein JJ, Komajda M, Lopez-Sendon J, Mosca L, Tardif JC, Waters DD, Shear CL, Revkin JH, Buhr KA, Fisher MR, Tall AR, Brewer B. Effects of Torcetrapib in patients at high risk for coronary events. N Engl J Med 2007; 357: 2109-2122.

3 Stowasser M, Sharman J, Leano R, Gordon RD, Ward G, Cowley D, Marwick TH. Evidence for abnormal left ventricular structure and function in normotensive individuals with familial hyperaldosteronism Type 1. J Clin Endocrinol Metab 2005; 90: 5070-5076.

4 Milliez P, Girerd X, Plouin PF, Blacher J, Safar ME, Mourad JJ. Evidence for an increased rate of cardiovascular events in patients with primary aldosteronism. J Am Coll Cardiol 2005; 45: 1243-1248.

5 Vasan RS, Evans JC, Larson MG, Wilson PW, Meigs JB, Rifai N, Benjamin EJ, Levy D. Serum aldosterone and the incidence of hypertension in nonhypertensive persons. N Engl J Med 2004; 351: 33-41.

6 Funder JW, Pearce P, Smith R, Smith AI. Mineralocorticoid action: target-tissue specificity is enzyme, not receptor, mediated. Science 1988; 242: 583-585.

7 Edwards CR, Stewart PM, Burt D, Brett L, Mclntyre MA, Sutanto WS, de Kloet ER, Monder C. Localisation of 11 beta-hydroxysteroid dehydrogenase-tissue specific protector of the mineralocorticoid receptor. Lancet 1988; 2: 986-989.

8 Funder JW, Myles K. Exclusion of corticosterone from epithelial mineralocorticoid receptors is insufficient for selectivity of aldosterone action: in vivo binding studies. Endocrinol 1996; 137: 5264-5268.

9 Fjeld C, Birdsong W, Goodman R. Differential binding of NAD+ and NADH allows the transcriptional corepressor carboxyl-terminal binding protein to serve as a metabolic sensor. Proc Natl Acad Sci USA 2003; 100: 9202-9207.

10 Alzamora R, Michea L, Marusic ET. Role of 11 beta-hydroxysteroid dehydrogenase on nongenomic aldosterone effects in human arteries. Hypertens 2000; 35: 1099-1104.
11 Qin W, Rudolph AE, Bond BR, Rocha R, Blomme EA, Goellner JJ, Funder JW, McMahon EG. A transgenic model of aldosterone-driven cardiac hypertrophy and heart failure. Circ Res 2003; 93: 69-76.

12 Pitt B, Remme W, Zannad F, Neaton J, Martinez F, Roniker B, Bittman R, Hurley S, Kleiman J, Gatlin M. Eplerenone, a selective aldosterone blocker, in patients with left ventricular dysfunction after myocardial infarction. N Engl J Med 2003; 348: 1309-1321.

13 Mihaildou AS, Loan Le TY, Mardini M, Funder JW. Glucocorticoids activate cardiac mineralocorticoid receptors during experimental myocardial infarction. Hypertens 2009; 54: 1306-1312.

14 Forrest MJ, Bloomfield D, Briscoe RJ, Brown PN, Cumiskey AM, Ehrhart J, Hershey JC, Keller WJ, Ma X, McPherson HE, Messina E, Peterson LB, Sharif-Rodriguez W, Siegl PK, Sinclair PJ, Sparrow CP, Stevenson AS, Sun SY, Tsai C, Vargas H, Walker III M, West SH, White V, Woltmann RF. Torcetrapib-induced blood pressure elevation is independent of CETP inhibition and is accompanied by increased circulating levels of aldosterone. Br J Pharmacol 2008; 154: 1465-1473.

$15 \mathrm{Hu}$ X, Dietz JD, Xia C, Knight DR, Loging WT, Smith AH, Yuan H, Perry DA, Keiser J. Torcetrapib induces aldosterone and cortisol production by an intracellular calcium-mediated mechanism independently of cholesteryl ester transfer protein inhibition. Endocrinology 2009; 150: 2211-2219. San Francisco, June 2008, p. 38

16 Capponi AM, Clerk RG, Campos L, Perez A, Garriz JMR, Maugeais C, Niesor EJ. No increase in the in vitro production of aldosterone on the expression of CYP $11 \beta 2$ with the CETP modulation dalcetrapib (R04607381/JTT-70S), in contrast with Torcetrapib. Circ 2008; 118: S452-S452.

17 Funder JW. The off-target effects of Torcetrapib. Endocrinol 2009; 150: 221-229.

18 Manunta P, Ferrandi M, Bianchi G, Hamlyn JM. Endogenous ouabain in cardiovascular function and disease. J Hypertens 2009; 27: 9-18.

19 Zhang J, Lee MY, Cavalli M, Chen L, Berra-Romani R, Balke CW, Bianchi G, Ferrari P, Hamlyn JM, Iwamoto T, Lingrel JB, Matteson DR, Wier WG, Blaustein MP. Sodium pump $\alpha 2$ subunits control myogenic tone and blood pressure in mice. J Physiol 2005; 569: 245-256.

20 Dostanic-Larson I, Van Huysse JW, Lorenz JN, Lingrel JB. The highly conserved cardiac glycoside binding site of $\mathrm{Na}$, K-ATPase plays a role in blood pressure regulation. Proc Natl Acad Sci USA 2005; 102: 15845-15850.

21 Levy D, Rocha R, Funder JW. Distinguishing the antihypertensive and electrolyte effects of eplerenone. J Clin Endocrinol Metab 2004; 89: 2736-2740. 